\title{
A DIGITAL SIGNAL PROCESSING PERSPECTIVE ON A STABILITY TEST OF LINEAR SYSTEM TRANSFER FUNCTIONS
}

\author{
Tania Stathaki and Anthony G. Constantinides \\ Signal Processing and Digital Systems Section \\ Department of Electrical and Electronic Engineering \\ Imperial College, United Kingdom
}

\begin{abstract}
The stability of a linear transfer function is fundamental in its real time realisation. Several tests have been developed in the past to test whether a given transfer function is stable. Invariably these tests rely on either a direct use of a Hurwitz test or some convenient modification of it such as the Schur-Cohen test or the Jury-Marden test. A new test is proposed in this contribution which does not rely on the same principles but on the Cauchy integral, and its implementation in practice requires merely the use of the Fast Fourier Transform. The test is convenient for real time implementation.
\end{abstract}

\section{INTRODUCTION}

The stability of a given transfer function $H(z)=N(z) / F(z)$ is of crucial significance in the realisation of the digital signal processing operation that it represents. As a result considerable effort has been devoted in the past to the problem of determining whether the given transfer function $H(z)$ is stable [1],[4],[7],[9],[10]. The condition for stability requires that the denominator $F(z)$ should have no zeros on, and outside $C:|z|=1$. In principle, therefore, one can test for stability by finding the zeros of $F(z)=0$, and testing whether they are all of modulus less than unity. However, when the order of the polynomial $F(z)$ is large or some zeros are very close to one another, root finding procedures would inevitably fail. At any rate it is not the actual position of the zeros of $F(z)$ that is required but rather whether they all share the property of having their modulus less than unity. Hence root finding techniques when they do work produce results that are more complex than those required by the question regarding stability.

Classical stability criteria that have been used extensively in the past are the Routh-Hurwitz and Nyquist stability criteria. A related and equally useful stability criterion that has been used extensively in discrete systems and digital filters is the Jury-Marden criterion [1],[4]. A new test is proposed in this contribution which does not rely on the same principles but on the Cauchy integral, and its implementation in practice requires merely the use of the Fast Fourier Transform. It is also eminently applicable to real time implementation.

\section{PRELIMINARIES}

We use the logarithmic residue of the polynomial $F(z)$ to compute a set of parameters indicative of the stability of $F(z)$. To this end, we set

$$
F(z)=z^{n}+p_{1} z^{n-1}+\ldots+p_{n}=\prod_{i=1}^{n}\left(z-r_{i}\right)
$$

The zeros of $F(z)$ may belong to any one of the following four classes:

(i) all $r_{i}$ have a modulus less than 1

(ii) all $r_{i}$ have a modulus greater than 1

(iii) some $r_{i}$ have a modulus less than 1 while the others are of modulus greater than 1

(iv) Anyone of the above with the addition that some $r_{i}$ have a modulus equal to unity.

We employ the following notation:

- $r_{j}=r_{j \text { in }}$ if the root $r_{j}$ is inside the unit circle.

- $r_{j}=r_{j o u t}$ if the root $r_{j}$ is outside the unit circle.

- $r_{j}=r_{j 0}$ if the root $r_{j}$ is on the unit circle.

Thus we can write

$$
\begin{aligned}
& F(z)=\prod_{i=1}^{n}\left(z-r_{i}\right)= \\
& {\left[\prod_{j}\left(z-r_{j \mathrm{in}}\right)\right]\left[\prod_{j}\left(z-r_{j \mathrm{out}}\right)\right]\left[\prod_{j}\left(z-r_{j \mathrm{o}}\right)\right]} \\
& \text { or } F(z)=F_{\min }(z) F_{\max }(z) F_{\mathrm{o}}(z)
\end{aligned}
$$

where the minimum phase part of $F(z), F_{\min }(z)$, is the polynomial that contains all the roots of $F(z)$ which are inside the unit circle and the maximum phase part of $F(z), F_{\max }(z)$, is the polynomial that contains all the roots of $F(z)$ 
which are outside the unit circle. The factor $F_{0}(z)$ contains all roots that are on the unit circle.

We define the parameters $\left\{S_{m}\right\}$ as follows [11]

$$
S_{m}=r_{1}^{m}+r_{2}^{m}+\cdots+r_{n}^{m}=\sum_{i=1}^{n} r_{i}^{m}
$$

From the definition of $S_{m}, m \in N$, of a polynomial it is evident that:

$$
S_{m}^{F(z)}=S_{m}^{F_{\min }(z)}+S_{m}^{F_{\max }(z)}+S_{m}^{F_{\mathrm{o}}(z)}
$$

We calculate the contour integral [2]-[4],[11]

$\frac{1}{2 \pi \mathrm{j}} \oint_{|z|=1} \frac{d}{d z}[\ln F(z)] z^{m} d z=\frac{1}{2 \pi \mathrm{j}} \oint_{|z|=1} \frac{F^{\prime}(z)}{F(z)} z^{m} d z$

$=\frac{1}{2 \pi \mathrm{j}} \oint_{z i=1}\left[\left(\sum_{j} \frac{1}{\left(z-r_{j \mathrm{in}}\right)}\right)\right.$

$\left.+\left(\sum_{j} \frac{1}{\left(z-r_{\text {jout }}\right)}\right)+\left(\sum_{j} \frac{1}{\left(z-r_{\text {jo }}\right)}\right)\right] z^{m} d z$

To evaluate the above integral we replace $z$ with $z=e^{\mathrm{j} \theta}$ and therefore we have for the polynomial

$$
F\left(e^{\mathrm{j} \theta}\right)=e^{\mathrm{j} n \theta}+p_{1} e^{\mathrm{j}(n-1) \theta}+\ldots+p_{n-1} e^{\mathrm{j} \theta}+p_{n}
$$

We now take $N$ points on the unit circle equally distributed to obtain the discrete values of $\theta$,

$$
\theta_{k}=\frac{2 \pi}{N} k, k=0,1, \ldots, N-1
$$

and hence

$$
\begin{aligned}
& \left.F(z)\right|_{z=e^{\mathrm{j} \theta_{k}}}=\left[\sum_{i=0}^{n} p_{i} e^{\mathrm{j}(n-i) \frac{2 \pi}{N} k}\right] \Rightarrow \\
& \left.F(z)\right|_{z=e^{\mathrm{j} \theta_{k}}}=e^{\mathrm{j} n \frac{2 \pi}{N} k}\left[\sum_{i=0}^{n} p_{i} e^{-\mathrm{j} i \frac{2 \pi}{N} k}\right]
\end{aligned}
$$

The expression inside the brackets is the Discrete Fourier Transform of the sequence $\left\{p_{i}\right\}, \quad i=0,1, \ldots, n$.

For the derivative $\frac{d}{d z}[F(z)]$ we have that

$$
\begin{aligned}
& \frac{d}{d z}\left[\left.F(z)\right|_{z=e^{j \theta}}=n e^{\mathrm{j}(n-1) \theta}+\right. \\
& (n-1) p_{1} e^{\mathrm{j}(n-2) \theta}+\ldots+2 p_{n-2} e^{\mathrm{j} \theta}+p_{n-1}
\end{aligned}
$$

Therefore, we have

$$
\begin{aligned}
& \frac{d}{d z}\left[\left.F(z)\right|_{z=e^{\mathrm{j} \theta_{k}}}=\left[\sum_{i=0}^{n-1}(n-i) p_{i} e^{\mathrm{j}(n-i-1) \frac{2 \pi}{N} k}\right]\right. \\
& \frac{d}{d z}\left[\left.F(z)\right|_{z=e^{\mathrm{j} \theta_{k}}}=e^{\mathrm{j}(n-1) \frac{2 \pi}{N} k\left[\sum_{i=0}^{n-1}(n-i) p_{i} e^{-\mathrm{j} i \frac{2 \pi}{N} k}\right]}\right.
\end{aligned}
$$

The expression inside the brackets is now the Discrete Fourier Transform of the sequence $\left\{(n-i) p_{i}\right\}, \quad i=0,1, \ldots, n-1$

so that

$$
\begin{aligned}
& \left.\frac{F^{\prime}(z)}{F(z)}\right|_{z=e^{\frac{j}{N} \frac{2 \pi}{N} k}} e^{j \frac{2 \pi}{N} k m}= \\
& \frac{D F T\left\{n,(n-1) p_{1}, \ldots, p_{n-1}\right\}(k)}{D F T\left\{1, p_{1}, \ldots, p_{n-1}, p_{n}\right\}(k)} e^{j \frac{2 \pi}{N} k(m-1)}
\end{aligned}
$$

If we choose $N$ such as $N=2^{M}$, where $M$ is a positive integer, then instead of a DFT we can use the computationally efficient Fast Fourier Transform (FFT)

$$
\begin{aligned}
& \left.\frac{F^{\prime}(z)}{F(z)}\right|_{z=e^{j} \frac{2 \pi}{2^{M} k}} e^{j \frac{2 \pi}{2^{M}} k m}= \\
& \frac{F F T}{F F T}\left\{\frac{\left.n,(n-1) p_{1}, \ldots, p_{n-1}\right\}(k)}{\left\{1, p_{1}, \ldots, p_{n-1}, p_{n}\right\}(k)} e^{j \frac{2 \pi}{2^{M} k(m-1)},}\right.
\end{aligned}
$$

Thus, in practice, the calculation of the contour integral (2) requires two FFT transforms and one IFFT transform [5],[6],[8]. Let us consider now the following two cases.

\subsection{The polynomial $F(z)$ has no zeros on the unit circle}

The terms containing $r_{j 0}$ are missing in equation (3) for this case. From the Cauchy's integral theorem we find that

$\frac{1}{2 \pi \mathrm{j}} \oint_{z \mid=1} \frac{z^{m} F^{\prime}(z)}{F(z)} d z= \begin{cases}S_{\text {min }}, & m \geq 1 \\ n_{\text {in }} & m=0 \\ -S_{-m \max } & m \leq-1\end{cases}$

where $n_{\text {in }}$ is the number of zeros inside the unit circle. To compute the above integrals we use approximations through summations based on the FFT as described earlier. Obviously the number of DFT bins $2^{M}$ must be greater than the degree of the polynomial $n$, otherwise we cannot express the polynomials uniquely.

\subsection{The polynomial $F(z)$ has at least one pair of complex conjugate zeros on the unit circle}

In this case, it follows from equation (3) and Cauchy's integral theorem that

$\frac{1}{2 \pi \mathrm{j}} \oint_{z=1} \frac{z^{m} F^{\prime}(z)}{F(z)} d z= \begin{cases}S_{m \min }+\frac{S_{m o}}{2} & m \geq 1 \\ n_{\mathrm{in}}+\frac{n_{\mathrm{o}}}{2} & m=0 \\ -S_{-m \max }-\frac{S_{m 0}}{2} & m \leq-1\end{cases}$

The summation through the FFT's must be examined more closely. The approximation of the integral is

$\frac{1}{2^{M}} \sum_{k=0}^{2^{M}-1}\left[\left(\sum_{j} \frac{1}{\left(z_{k}-r_{j \mathrm{in}}\right)}\right)+\right.$ 
$\left.\left(\sum_{j} \frac{1}{\left(z_{k}-r_{\text {jout }}\right)}\right)+\left(\sum_{j} \frac{1}{\left(z_{k}-r_{j 0}\right)}\right)\right]_{z=z_{k}} e^{j \frac{2 \pi}{2^{M}} k} e^{\frac{2 \pi}{2^{M} k m}}$

If $r_{i}=e^{j \psi}$ is one of the zeros on the unit circle then its contribution to the summation for $m=0$ is:

$$
\begin{gathered}
\frac{1}{2^{M}} \sum_{k=0}^{2^{M}-1} \frac{1}{z_{k}-e^{\mathrm{j} \psi}} z_{k}=\frac{1}{2^{M}} \sum_{k=0}^{2^{M}-1} \frac{e^{\mathrm{j} \frac{2 \pi}{2^{M}} k}}{e^{\frac{2 \pi}{2^{M}} k}-e^{\mathrm{j} \psi}}= \\
\quad=\frac{1}{2}+\frac{\mathrm{j}}{2 \cdot 2^{M}} \sum_{k=0}^{2^{M}-1} \cot \left[\left(\Psi-\frac{2 \pi}{2^{M}} k\right) / 2\right]
\end{gathered}
$$

Similarly we have that

$$
\begin{aligned}
& \frac{1}{2^{M}} \sum_{k=0}^{2^{M}-1} \frac{1}{z_{k}-e^{-\mathrm{j} \psi} z_{k}} \\
& =\frac{1}{2}-\frac{\mathrm{j}}{2 \cdot 2^{M}} \sum_{k=0}^{2^{M}-1} \cot \left[\left(\psi+\frac{2 \pi}{2^{M}} k\right) / 2\right] \text { and hence } \\
& \frac{1}{2^{M}} \sum_{k=0}^{2^{M}-1} \frac{1}{z_{k}-e^{j \psi}} z_{k}+\frac{1}{2^{M}} \sum_{k=0}^{2^{M}-1} \frac{1}{z_{k}-e^{-\mathrm{j} \psi}} z_{k}=1
\end{aligned}
$$

There are two cases we need to consider here depending on the position of $\psi$ relative to the DFT points $\theta_{k}$

$$
\text { (a) } \psi=\psi_{k} \neq \frac{2 \pi}{2^{M}} k
$$

It is evident from the above analysis that the contribution of this zero to the summation on the unit circle, and its complex conjugate, is equal to 1. In that case the approximation of the integral by a summation gives the correct result.

(b) $\quad \psi=\psi_{k}=\frac{2 \pi}{2^{M}} k$

We have a specific $k$ in this case for which $\psi=\psi_{k}=\frac{2 \pi}{2^{M}} k$ and hence

$$
\sum_{k=0}^{2^{M}-1} \cot \left[\left(\psi \pm \frac{2 \pi}{2^{M}} k\right) / 2\right]=\infty
$$

Thus the summation gives an infinite answer, which is not the result expected from the integration. In this case the DFT-based approximation of the line integral fails. However the infinite value does indicate the evidence of a zero on $|z|=1$ and hence the unstable nature of the given polynomial.

From the above analysis the following points can be gathered synoptically:

If the function $F(z)=z^{n}+p_{1} z^{n-1}+\ldots+p_{n}$ has

- $n_{\text {in }}$ zeros inside the unit circle

- $n_{\mathrm{o}}$ zeros on the unit circle

- $n_{\text {out }}$ zeros outside the unit circle

and $M$ is a positive integer such that $2^{M} \gg n$ then (i) If there is no $k$ in the set $\left\{0,1, \ldots, 2^{M}-1\right\}$ such that $r_{k}=e^{\frac{j}{2^{M} k} k}$, then the DFT-based approximation of equation (2) yields approximately $S_{\mathrm{o}}=n_{\mathrm{in}}+\frac{n_{\mathrm{o}}}{2}$. (ii) If there is a $k\left\{0,1, \ldots, 2^{M}-1\right\}$ such that $r_{k}=e^{j \frac{2 \pi}{2^{M} k}}$, then the DFT-based computation of equation (2) yields an infinite result.

\subsection{Error Due To The Approximation of Integrals by Summations}

Let us suppose that we have one pair of zeros inside the unit circle. We wish to determine the error in approximating the integral by summations. Specifically we would like to know the value of the error $S_{0}$, where the zeros are located at $\rho e^{ \pm \mathrm{j} \phi}$

$\oint\left(\frac{1}{z-\rho e^{\mathrm{j} \phi}}+\frac{1}{z-\rho e^{-\mathrm{j} \phi}}\right) d z-$

$\frac{1}{2^{M}} \sum_{k=0}^{2^{M}-1}\left(\frac{e^{j \frac{2 \pi}{2^{M} k}}}{z_{k}-\rho e^{\mathrm{j} \phi}}+\frac{e^{\frac{j \pi}{2^{M}} k}}{z_{k}-\rho e^{-\mathrm{j} \phi}}\right)$

Let

$$
\begin{aligned}
& A=\sum_{k=0}^{2^{M}-1}\left(\frac{e^{\mathrm{j} \frac{2 \pi}{2^{M}} k}}{z_{k}-\rho e^{\mathrm{j} \phi}}+\frac{e^{\mathrm{j} 2^{2^{M}}}}{z_{k}-\rho e^{-\mathrm{j} \phi}}\right) \\
& =\frac{2^{M}}{1-\rho^{2^{M}} e^{\mathrm{j} 2^{M} \phi}}+\frac{2^{M}}{1-\rho^{2^{M}} e^{-\mathrm{j} 2^{M} \phi}}= \\
& =2 \cdot 2^{M} \frac{1}{1+\rho^{2^{M}}\left(\frac{\rho^{2^{M}}-\cos \left(2^{M} \phi\right)}{1-\rho^{2^{M}} \cos \left(2^{M} \phi\right)}\right)}
\end{aligned}
$$

Since $\rho<1$, it follows that $\left|\frac{\rho^{2^{M}}-\cos \left(2^{M} \phi\right)}{1-\rho^{2^{M}} \cos \left(2^{M} \phi\right)}\right|<1$.

Therefore in the limit,

$$
\frac{A}{2^{M}} \rightarrow \frac{2 \cdot 2^{M}}{2^{M}} \text { as } 2^{M} \rightarrow+\infty \text {. }
$$

The required value is actually 2 , and hence the error $\varepsilon$ is given by

$$
\varepsilon=2-2 \frac{1}{1+\rho^{2^{M}}\left(\frac{\rho^{2^{M}}-\cos \left(2^{M} \phi\right)}{1-\rho^{2^{M}} \cos \left(2^{M} \phi\right)}\right)}
$$

\section{A NEW STABILITY TEST}

The above results enable us to formulate a simple stability test as follows. If the denominator of the 
given transfer function is $F(z)$ the requirement for stability is that it should have no zeros outside and on the exist circle $C:|z|=1$. However, if we take as the initial polynomial to be tested not $F(z)$ but $D(z)=z^{n} F\left(z^{-1}\right)$ then we have considerable simplification. The associated polynomial $D(z)$ has the following properties. Let

$$
F(z)=z^{n}+p_{1} z^{n-1}+p_{2} z^{n-2}+\ldots+p_{n}
$$

Then

$$
D(z)=p_{n} z^{n}+p_{n-1} z^{n-1}+\ldots+p_{1} z+1
$$

The zeros of $D(z)$ are in the complementary regions to those of $F(z)$ with respect to the unit circle on the z-plane, except for those zeros on the contour $C:|z|=1$ which for real polynomials remain at the same locations. Thus if $F(z)$ is of degree $n$ then the requirement for stability is that the inverse polynomial $D(z)=z^{n} F\left(z^{-1}\right)$ should have no zeros within $C:|z|=1$.

Thus if the given transfer function is stable then

$$
\oint_{c} \frac{D^{\prime}(z)}{D(z)} z^{m} d z=0
$$

In practice, of course, the integration may be implemented through the use of FFTs as described above.

Thus for $z=e^{j \theta}$

$$
\oint_{c} \frac{D^{\prime}(z)}{D(z)} z^{m} d z=\int_{-\pi}^{\pi} \frac{D^{\prime}\left(e^{\mathrm{j} \theta}\right)}{D\left(e^{\mathrm{j} \theta}\right)} \mathrm{j} e^{\mathrm{j}(m+1) \theta} d \theta=0
$$

and for discrete $\theta_{k}=\frac{2 \pi}{N} k \quad k=0,1, \ldots, N-1$ we obtain

$$
\frac{2 \pi}{N} \sum_{k=0}^{N-1}\left[\frac{D^{\prime}\left(e^{\mathrm{j} \theta_{k}}\right)}{D\left(e^{\mathrm{j} \theta_{k}}\right)}\right] e^{\mathrm{j}(m+1) \theta_{k}}=0
$$

Hence we need only take the inverse FFT of $\frac{D^{\prime}(z)}{D(z)}$ and examine it at the index value of 1 . If the result is other than zero then the given transfer function is unstable. In practice the result may not be exactly equal to zero even for stable transfer functions. However for large $N$, it will be a small number the integer part of which will be zero. Since the result is at best an integer, for the unstable case, any other numerical result with zero integer part is sufficient to indicate stability. The use of FFT to compute the integral, yields not only the required result, i.e. the number of zeros within the unit circle of the reciprocal polynomial to $F(z)$ which corresponds to $k=1$, but it also yields the sums of the powers of these zeros corresponding to the other values for the index $k$. Since there are no zeros of $F(z)$ within the unit circle these additional values will also be zero when $D(z)$ is stable. This provides additional checks on the stability.

\section{EXPERIMENTAL RESULTS}

The stability test has been applied to a very large number of stable and unstable polynomials of various order from 20 to 200 and it proved to be $100 \%$ successful.

\section{DISCUSSION}

The computational complexity of the test is associated with the FFT operations and the element by element division of two arrays in (4). Assuming the division operation to be of the same complexity as a complex multiplication we have a computational complexity of $\mathrm{O}\left(3 N \log _{2} N+N\right)$. This compares favourably with the Jury-Marden triangular array of complexity $\mathrm{O}[N(N+1) / 2]$.

\section{REFERENCES}

[1] Antoniou A., Digital Filters Analysis, Design, and Applications, McGraw-Hill, 1993.

[2] Borowski E. J. and J. M. Borwein, Dictionary in Mathematics, Collins, 1989.

[3] Henrici P., Applied and Computational Complex Analysis, Voll.1, New York: McGrawHill, 1986.

[4] Jury E. I., Theory and Application of zTransform Method, New York: Wiley 1964.

[5] Nikias C. L. and F. Liu, Bicepstrum on Computation Based on Second and Third Order Statistics with Applications", Proc. ICASSP 90, p. 2381, April 1990, Albuquerque NM.

[6] Nikias C. L. and A. Petropulu, Higher Order Spectra Analysis, Prentice Hall, 1993.

[7] Oppenheim A. V. and R. W. Schafer, Discrete Time Signal Processing, Englewood Cliffs, NJ: Prentice Hall, 1989.

[8] Polydoros A. and A. T. Fam, "The Differential Cepstrum Definition and Properties", Proc. IEEE Int. Symposium on Circuits and Systems, p. 77, April 1981.

[9] Rabiner L. R. and R. W. Schafer, Digital Processing of Speech Signals, Englewood Cliffs, NJ: Prentice-Hall, 1978.

[10] Snell R. C. and F. Milinazzo, "Formant Location From. LPC Analysis Data", IEEE Trans. on ASSP, Vol. 1, No. 2, April 1993.

[11]'Todhunter I., Theory of Equations, Mc Millan \& Co, London 1882. 